\title{
Transition Regime Analytical Solution to Gas Mass Flow Rate in a Rectangular Micro Channel
}

\author{
S Kokou Dadzie* and Nishanth Dongari ${ }^{\dagger}$ \\ *Mechanical and Aeronautical Engineering, Glyndŵr University, Mold Road, Wrexham LL11 2AW, UK \\ $\dagger$ Department of Mechanical and Aerospace Engineering, University of Strathclyde, Glasgow G1 1XJ, UK
}

\begin{abstract}
We present an analytical model predicting the experimentally observed gas mass flow rate in rectangular micro channels over slip and transition regimes without the use of any fitting parameter. Previously, Sone reported a class of pure continuum regime flows that requires terms of Burnett order in constitutive equations of shear stress to be predicted appropriately. The corrective terms to the conventional Navier-Stokes equation were named the ghost effect. We demonstrate in this paper similarity between Sone ghost effect model and newly so-called 'volume diffusion hydrodynamic model'. A generic analytical solution to gas mass flow rate in a rectangular micro channel is then obtained. It is shown that the volume diffusion hydrodynamics allows to accurately predict the gas mass flow rate up to Knudsen number of 5 . This can be achieved without necessitating the use of adjustable parameters in boundary conditions or parametric scaling laws for constitutive relations. The present model predicts the non-linear variation of pressure profile along the axial direction and also captures the change in curvature with increase in rarefaction.
\end{abstract}

Keywords: mass diffusion; volume diffusion hydrodynamics; microchannel gas flow; Knudsen paradox; pressure distribution; slip flows PACS: 47.61.-k, 47.61.Cb, 47.61.Fg

\section{INTRODUCTION}

Growing demands in microdevice technology involving the motion of fluids at micro- and nano-length scales open a new branch in fluid mechanics requiring investigation of fluid flows occurring exclusively in ultra-small devices. Examples of these mechanical systems include micro-pumps, heat exchangers, jet polishing/cutting systems and, more importantly, the entire range of Micro-Electro-Mechanical Systems (MEMS) encompassing their various bioengineering applications $[1,2,3]$.

Knudsen [4] experimental study, was among the first designed to report data pointing to anomalous behaviour of rarefied gas flow through capillaries. Many experiments have since confirmed Knudsen's initial observations [5, 6, 7]. Meanwhile, it is now widely accepted that the standard set of fluid mechanical equations, namely those due to NavierStokes-Fourier, are inapplicable. Among recent developments in predicting these phenomena, Gallis and Torczynski [8] have presented a direct Monte Carlo simulation (DSMC). Gorji et al. [9] obtained the mass flowrate by solving, numerically, a velocity-space stochastic equation addressing molecular motions. Veltzke and Thaming [10] pointed out that the mass flowrate in the slip flow regime can be accurately predicted by arguments of molecular spatial diffusion effects without using any fitting parameter.

Sone [11] suggested a correction to the standard Navier-Stokes equation, where the additional terms with characteristics of Burnett equation terms were called 'ghost effect terms'. This is because they affect flows of continuum regime despite being of high order in terms of Knudsen number classification. Meanwhile, existence of different averaging methods and the influence of molecular spatial stochasticity have been pointed out as means of obtaining a Burnett regime hydrodynamic model [12]. This model appears more consistent in terms of mechanical and thermodynamic properties than a traditional full Burnett equation obtained by Chapman-Enskog series expansions applied to Boltzmann kinetic equation [12]. In the present paper we derive an analytical solution to mass flow rate in microchannels using this new emerging hydrodynamics and compare results with experimental data. 


\section{A CONSISTENT VOLUME DIFFUSION HYDRODYNAMIC MODEL: SONE GHOST EFFECT}

Appearing in the following equations is the material derivative defined as $D / D t=\partial / \partial t+U_{m} \cdot \nabla$, where $t$ is the time variable and $U_{m}$ represents mass-based average velocity: that is a flow macroscopic velocity as seen in a conventional continuity equation. A volume diffusion hydrodynamic model consistent with mechanical and thermodynamic principles is written [12]:

$$
\begin{gathered}
\frac{D \rho}{D t}=-\rho \nabla \cdot U_{m}, \\
\rho \frac{D U_{m}}{D t}=-\nabla \cdot \Pi, \\
\rho \frac{D}{D t}\left[\frac{1}{2} U_{m}^{2}+e_{i n}\right]=-\nabla \cdot\left[\Pi \cdot U_{v}\right]-\nabla \cdot J_{u},
\end{gathered}
$$

that is closed with

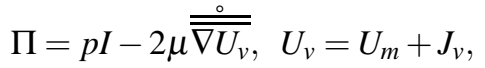

where

$$
J_{v}=\frac{\kappa_{m}}{\rho} \nabla \rho, J_{u}=-\kappa_{h} \nabla T
$$

and

$$
\stackrel{\stackrel{\circ}{\overline{\nabla U_{v}}}}{=} \frac{1}{2}\left(\nabla U_{v}+\overline{\nabla U_{v}}\right)-\frac{1}{3} \nabla \cdot U_{v} I .
$$

The single bar over the velocity gradient in this last equation denotes the transpose operator, with $I$ the idemfactor. In the above set of equations (1) to (5): $\rho$ is the mass density, $p$ the pressure, $T$ the temperature, and $e_{i n}$ the fluid's massspecific internal energy density, the latter related to the temperature by $e_{i n}=(3 / 2) R T$, with $R$ the specific gas constant. Velocity $U_{v}$ appearing in expression of the shear stress is the volume velocity: that is a macroscopic velocity based on averaging method that accounts for the fluid molecular spatial distributions and not only their masses. Therefore $J_{v}$ is a volume diffusion flux that appears as we distinguish volume averaging from mass or gravimetric averaging [13]. Presence of flux $J_{v}$ in the shear stress makes the above hydrodynamic model of Burnett level. Indeed, using the ideal gas law written in the form

$$
\frac{\nabla p}{p}=\frac{\nabla \rho}{\rho}+\frac{\nabla T}{T}
$$

volume diffusion momentum equation (2) becomes

$$
\rho \frac{D U_{m}}{D t}=-\nabla \cdot\left[p I-2 \mu \overline{\overline{\nabla U_{m}}}+2 \mu \kappa_{m} \overline{\overline{\nabla \nabla \ln T}}\right],
$$

if local relative pressure variations are neglected. In equation (8), we observe that the third term involving temperature gradients on the right-hand-side, is a thermal stress term that is obtained traditionally at the second order in ChapmanEnskog expansion; so it is a Burnett shear stress term. This term is identical to corrective terms to the Navier-Stokes equation called "ghost effect terms": conveying that this is a higher order Knudsen number term, which is found to influence flows in the pure hydrodynamics or pure continuum regime. Derivation of volume diffusion hydrodynamic set (1) to (5), does not involve any small parameter expansion procedure. Consequently, within this set of equations, the volume diffusion presumably may have influence at any Knudsen number order; a situation that appears to corroborate with Sone ghost effects.

While a classical set of full Burnett hydrodynamic equations derived using Chapman-Enskog expansion series are known to have several mechanical and thermodynamic inconsistencies [14], the above set of equations (1) to (5) satisfies a series of mechanical principles and is compatible with linear irreversible thermodynamics [12, 15]. Phenomenological transport coefficients involved consist of: $\mu$ the fluid dynamic viscosity, $\kappa_{m}=k /\left(c_{p} \rho\right)$ the volume diffusivity coefficient, and $\kappa_{h}=k c_{v} / c_{p}$, with $k$ the Fourier thermal conductivity, namely the conductivity appearing in the Prandtl number, with $c_{v}$ and $c_{p}$ the specific heat conductivities [see 16, section5.4.1] and also [see 15, section 5.2]. 


\section{ANALYTICAL SOLUTION}

We consider a steady-state isothermal pressure-driven flow occurring in a rectangular microchannel. The streamwise flow coordinate variable is denoted $x$ and the wall normal coordinate is denoted $y$. The height and width of the channel are denoted respectively by $h$ and $w$, wherein $w>>h$ such that the flow may be supposed two-dimensional. The rectangular channel height-to-length ratio $h / L$ is assumed to be small to neglect the inlet and outlet effects. Velocity component, $U_{m}=U_{m}(x, y)$ is restricted to the streamwise direction and is a function of $x$ and $y$. Disregarding energy equation, continuity and volume diffusion momentum equations (1) and (2) can be written as :

$$
\begin{gathered}
\nabla \cdot\left[\rho U_{m}\right]=0, \\
\nabla \cdot\left[\rho U_{m} U_{m}\right]=-\nabla p-\nabla \cdot \Pi_{v} .
\end{gathered}
$$

In terms of boundary conditions, impenetrability of mass at the channel walls requires normal component of mass velocity $U_{m}$ to vanish at $y= \pm h / 2$. In addition, we impose on the volume velocity a form of slip condition so that all boundary conditions are written:

$$
u_{m_{y}}(x, \pm h / 2)=0
$$

and

$$
u_{v_{x}}(x, \pm h / 2)=J_{v_{x}}(x, \pm h / 2)=\mp \lambda_{o} \rho_{o} \frac{1}{\rho}\left[\frac{\partial u_{v_{x}}}{\partial y}\right]_{y=h / 2}=\mp \lambda_{o} \rho_{o} \frac{1}{\rho}\left[\frac{\partial u_{m_{x}}}{\partial y}\right]_{y=h / 2},
$$

In boundary conditions (11) and (12), subscript $x$ and $y$ refer to components in $x$ and $y$ directions, respectively. Subscript ' $o$ ', refers to the channel outlet, which is simply used here as a convenient arbitrary reference. Furthermore, $\lambda_{o}$ is the gas mean free path at the channel outlet. Note that equation (12) is not a standard slip condition, as the equation can be viewed as a constitutive equation for the volume flux $J_{v}$ at the boundary when $u_{m_{x}}=0$ (i.e., when the no-slip boundary condition is imposed on the mass velocity).

A solution method starts with continuity and momentum equations (9) and (10), reformulated as:

$$
\begin{gathered}
\rho \frac{\partial u_{m_{x}}}{\partial y}+\frac{\partial}{\partial x}\left[\rho u_{v_{x}}\right]-\frac{k}{c_{p}} \frac{d^{2} \ln \rho}{d x^{2}}=0, \\
\mu \frac{\partial^{2} u_{v_{x}}}{\partial y^{2}}=\frac{d p}{d x} .
\end{gathered}
$$

The solution of equation (14) satisfying boundary condition (12) is

$$
u_{v_{x}}=\frac{1}{8 \mu} \frac{d p}{d x}\left(4 y^{2}-h^{2}-8 \mu E \frac{1}{\rho}\right)
$$

in which we denote $E=h^{2} K n_{o} /\left(2 v_{o}\right)$, wherein $v_{o}$ denotes the kinematic viscosity at the outlet, and $K n_{o}=\lambda_{o} / h$ is the outlet Knudsen number. Substituting (15) into (13) and solving the resulting expression for $u_{m_{x}}$, by subjecting to the boundary condition (11) results in

$$
u_{m_{x}}=-\frac{1}{8 \mu}\left(\frac{4}{3} y^{3}-h^{2} y\right) \frac{1}{\rho} \frac{d}{d x}\left[\frac{\rho d p}{d x}\right]+E y \frac{1}{\rho} \frac{d^{2} p}{d x^{2}}+y \frac{k}{c_{p} \rho} \frac{d^{2} \ln \rho}{d x^{2}}+C(x),
$$

where the integration constant $C(x)$ is a function only of $x$. However, application of boundary conditions (12) requires that $C(x)=0$ as a result of symmetry. The preceding equations furnish the pressure distribution in accordance with the following scheme. Evaluate equation (16) at $y= \pm h / 2$, and use boundary condition (12) to obtain

$$
\frac{1}{12} h^{2} \frac{d}{d x}\left[\frac{\rho d p}{d x}\right]+E \frac{d^{2} p}{d x^{2}}+\frac{k}{c_{p}} \frac{d^{2} \ln \rho}{d x^{2}}=0 .
$$

Eliminate the density in equation (17) in favour of the pressure via use of the ideal gas law $p=\rho R T$, and subsequently use the identity $p d p / d x=(d / d x)\left(p^{2} / 2\right)$ to obtain

$$
\frac{d}{d x^{2}}\left[p^{2}+\frac{24 \mu R T}{h^{2}}\left(E p+\frac{k}{c_{p}} \ln p\right)\right]=0 .
$$


Integration of equation (18) followed by rearrangement yields

$$
\left(\frac{p}{p_{o}}\right)^{2}+F \frac{p}{p_{o}}+G\left(\ln \left(\frac{p}{p_{o}}\right)+\ln p_{o}\right)=C \frac{x}{L}+D,
$$

where

$$
F=\frac{24 \mu R T}{h^{2} p_{o}} E \text { and } G=\frac{24 \mu R T}{h^{2} p_{o}^{2}} \frac{k}{c_{p}} .
$$

Alternatively, in terms of the Knudsen and Prandtl numbers,

$$
K n_{o}=\frac{k_{\lambda}}{h} \frac{\mu}{p_{o}} \sqrt{2 R T}, \quad \operatorname{Pr}=c_{p} \mu / k, \quad F=12 K n_{o}, \quad G=\frac{24}{\operatorname{Prk}_{\lambda}^{2}} K n_{o}^{2},
$$

where $k_{\lambda}=\sqrt{\pi / 2}$ is a coefficient associated with the definition of the mean free path as related to the choice of molecular collision model. Equation (19) is the pressure distribution, in which constants $C$ and $D$ are determined from knowledge of the channel inlet and outlet pressures, $p(x=0)=p_{i}$ and $p(x=L)=p_{o}$. This furnishes expressions

$$
C=-\left[\left(P^{2}-1\right)+F(P-1)+G \ln P\right], \quad D=P^{2}+F P+G\left(\ln P+\ln p_{o}\right),
$$

where $P=p_{i} / p_{o}$, denotes the inlet/outlet pressure ratio. The mass flowrate through the channel is given by the formula

$$
\dot{M}=w \int_{-h / 2}^{h / 2} \rho U_{m} d y=\text { const. }
$$

Using $U_{m}=U_{v}-J_{v}$, with equations (15) and (5) we have that

$$
u_{m_{x}}=\frac{1}{8 \mu} \frac{d p}{d x}\left(4 y^{2}-h^{2}-8 \mu E \frac{1}{\rho}\right)-\frac{k}{c_{p} \rho} \frac{d \ln \rho}{d x} .
$$

Multiply equation (24) by $\rho$ and subsequently eliminate $\rho$ in favor of $p$ on the right-hand side. Introduction of the resulting expression into equation (23) yields

$$
\dot{M}=-\frac{w h^{3}}{24 \mu R T} \frac{d}{d x}\left[p^{2}+\frac{24 \mu R T}{h^{2}}\left(E p+\frac{k}{c_{p}} \ln p\right)\right] .
$$

The bracketed term in equation (25) is seen to be identical to the bracketed term in equation (18). It follows that

$$
\dot{M}=-\frac{w h^{3} p_{o}^{2}}{24 L \mu R T} C,
$$

where $C$ is the constant given by equation (22). Thus, the mass flow rate is given explicitly in terms of the parameters characterizing the problem as

$$
\dot{M}=\frac{w h^{3} p_{0}^{2}}{24 L \mu R T}\left[\left(P^{2}-1\right)+12 K n_{o}(P-1)+\frac{24}{\operatorname{Prk}_{\lambda}^{2}} K n_{o}^{2} \ln P\right] .
$$

Equation (27) has a structure frequently obtained when slip boundary conditions are used [17]. However, our goal in this investigation is to demonstrate that, due to the use of volume diffusive flux, equation (27) can reproduced faithfully the experimental data without the use of any fitting parameter.

Indeed, in the course of gas-kinetic molecular description of the hydrodynamic set of equations (1)-(5), volume diffusion is a manifestation of the molecular-level spatial diffusion process that used to be ignored [18]. This, in turn, generates a second level of non-equilibrium scaling beyond, or parallel to, traditional Knudsen number scaling [12]. Veltzke and Thaming [10] demonstrated in this context that volume diffusivity coefficient depends not only on the properties of the gas but also on the channel geometry. This leads us to identify a volume diffusivity coefficient for the rectangular channel corrected by the channel dimensions as, $\kappa_{m}^{*}=\kappa_{m} L / w$. As thermodynamic expression of the volume diffusivity coefficient is $\kappa_{m}=k /\left(c_{p} \rho\right)$, this geometrical correction only affects the Prandtl number coefficient 
TABLE 1. Summary of fluid properties and physical coefficients used in figure 1

\begin{tabular}{cccc}
\hline$w$ & $L$ & $h$ & $P r$ \\
\hline $4.92 \times 10^{-4}[\mathrm{~m}]$ & $9.39 \times 10^{-3}[\mathrm{~m}]$ & $9.38 \times 10^{-6}[\mathrm{~m}]$ & 0.67 \\
\hline$\mu$ & $k_{\lambda}$ & $S c$ & $P$ \\
\hline $1.97513 \times 10^{-5}[\mathrm{~Pa} \cdot \mathrm{s}]$ & $\sqrt{\pi / 2}$ & 0.67 & 5 \\
\hline
\end{tabular}

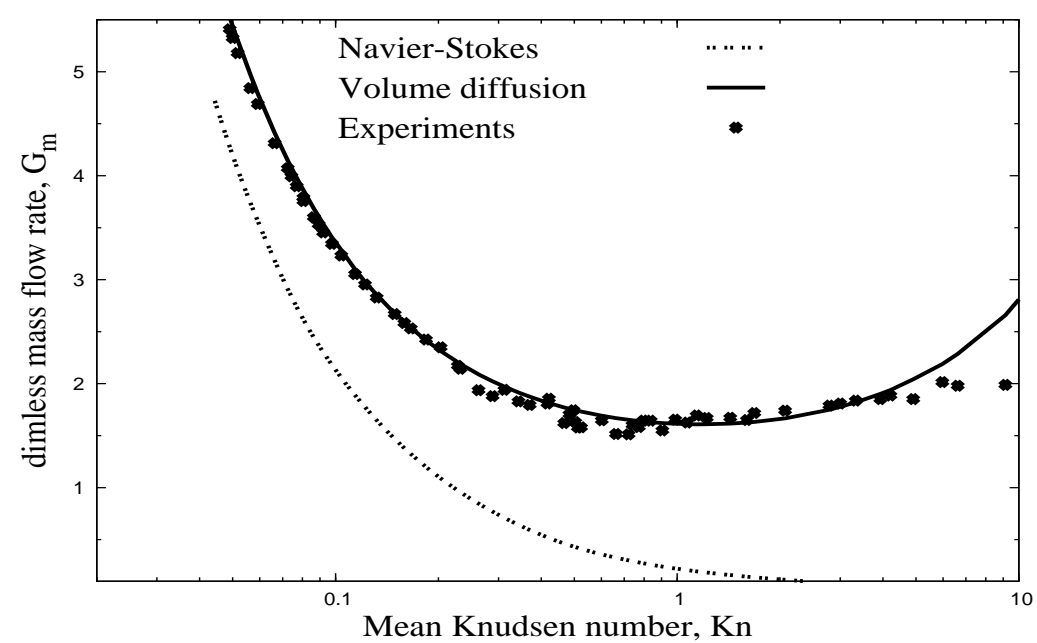

FIGURE 1. Mass flow rate as predicted by volume diffusion hydrodynamics compared with experimental data

by a factor $L / w$ in the final pressure distribution equation (19), and the mass flow rate equation (27). Full expression of the micro gas mass flow rate in rectangular channel using the volume diffusion momentum equation is therefore finally given by

$$
\dot{M}=\frac{w h^{3} p_{0}^{2}}{24 L \mu R T}\left[\left(P^{2}-1\right)+12 K n_{o}(P-1)+\frac{24 w}{P r L k_{\lambda}^{2}} K n_{o}^{2} \ln P\right],
$$

in which, there appear only physical properties with no coefficient playing a fitting role such as a slip coefficient. These physical properties in the case of Ewart et al. [7] experimental data for gaseous helium are summarized in table 1. A full comparison between predictions by equation (28) and experiments are seen in figure 1, plotted in the form of dimensionless flowrate, $G_{m}$, vs the mean Knudsen number that are defined by

$$
K n[\text { mean }]=\frac{k_{\lambda}}{h} \frac{\mu}{\frac{p_{i}+p_{0}}{2}} \sqrt{2 R T}, \quad G_{m}=\dot{M} *\left(\frac{w h^{2}}{L \sqrt{2 R T}}\left(p_{\text {in }}-p_{\text {out }}\right)\right)^{-1} .
$$

From the figure, the volume diffusion model, i.e. equation (28), agrees with experimental data up to a Knudsen number of about 5 with all parameters possessing clear physical meanings as given in table 1 and a viscosity coefficient having its appropriate experimental value. This agreement can be said an excellent achievement considering the fact that conventional Navier-Stokes modified by slip boundary corrections, gives agreement with the data within the slip but not deep into the transition regime as the present volume diffusion model does.

Figure 2 presents a comparison of normalized streamwise pressure distributions against the experimental data of Pong et al. [19] at various pressure ratios $(P)$. Here, pressure is normalized with the outlet pressure of the channel, and the pressure ratio is defined as the ratio of inlet to outlet pressures. The measurements by Pong et al. [19] were made by embedding measurement ports in a microchannel in which pressure transducers were mounted. The working gas was nitrogen and the outlet Knudsen number was 0.044 . So theoretical graphs are plotted using pressure distribution equation (19) including nitrogen Prandtl number of $P r=0.72$ and the geometrical correction factor $L / w$ from the volume diffusivity coefficient. The comparison between the present volume diffusion model and the data is good. 


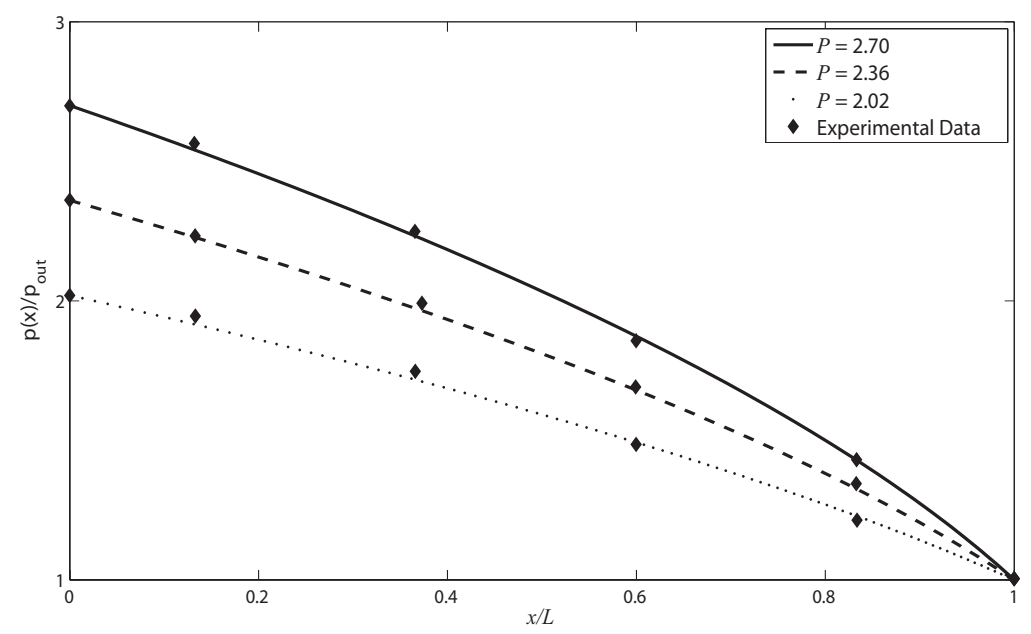

FIGURE 2. Pressure distribution as predicted by volume diffusion model compared with experimental data of Pong et al. [19] for an outlet Knudsen number of 0.044 and various pressure ratios $(P)$.

(a)

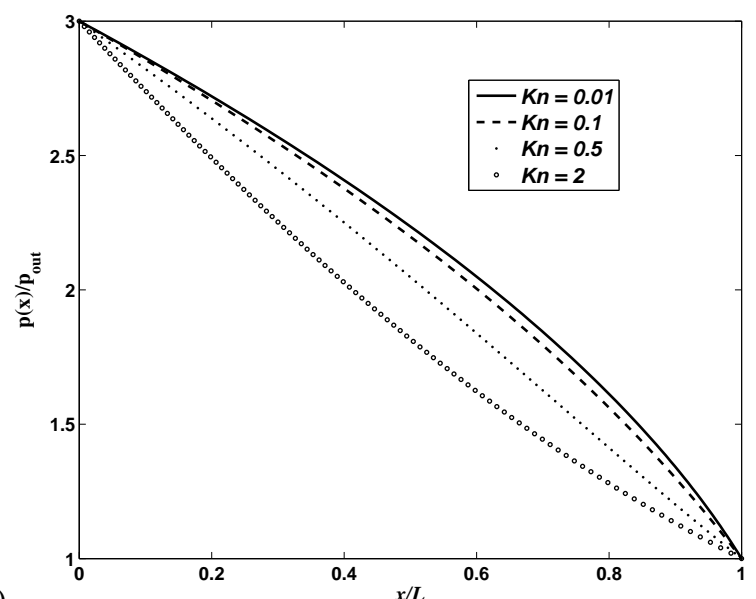

FIGURE 3. (a) Normalized pressure distribution along the streamwise direction for various Knudsen numbers, (b) dimensionless deviation of the pressure distribution from the linear pressure distribution as a function of the dimensionless distance.

The volume diffusion effect on the streamwise pressure distribution can be assessed by varying the Knudsen number. Interesting results are observed as shown in the Fig. 3(a) for a pressure ratio of 3. The curvature of the pressure variation decreases with an increase in Knudsen number, and the pressure distribution becomes linear in the early transition regime. With further increases in Knudsen number the curvature changes from convex (in the continuum and early transition regimes) to concave (in the late transition regime). Change in curvature of pressure profile can also be captured by the second-order slip model [20], however the classical first-order slip model fails to predict this phenomena. Fig. 3(b) shows the dimensionless deviation of the streamwise pressure distribution from a linear pressure profile, as a function of dimensionless distance along the channel. With an increase in Knudsen number, the rarefaction effect tends to dominate compressibility effects, and the dimensionless deviation becomes zero at a Knudsen number of around 0.5. With further increases in Knudsen number, the dimensionless deviation changes from positive to negative value. In the slip flow regime, where compressibility effects are still dominant, the pressure profile is unsymmetrical with respect to the streamwise distance, but turns out to be quite symmetric in the transition regime at $K n=2$, where rarefaction/volume diffusion effects are dominant. 


\section{CONCLUSION}

In this paper we derived an analytical solution to Knudsen enhanced mass flowrate phenomena in micro gas channels using a volume diffusion hydrodynamic model. The solution equation is similar in form to that obtained using first and second order slip boundary conditions. However, with the volume diffusion approach and volume diffusivity coefficient that depends on the geometry of the channel, we obtain agreement with the experimental data up to Knudsen number of 5. It also allows the direct investigation of the non-linear pressure distribution, and predicts the change in curvature of the streamwise pressure profile with an increase in rarefaction (when the diffusive effects dominate the convective fluxes).

\section{ACKNOWLEDGMENTS}

ND would like to thank the European Community's Seventh Framework Programme FP7/2007-2013 under grant agreement ITN GASMEMS n 215504.

\section{REFERENCES}

1. G. Karniadakis, A. Beskok, and N. R. Aluru, Microflows and Manoflows: Fundamentals and simulations, Springer, 2005.

2. S. Colin, Microfluidics, ISTE, 2010.

3. G. Kandlikar, S. Garimella, D. Li, S. Colin, and M. R. King, Heat transfer and fluid flow in minichannels and microchannels, Elsevier, 2006.

4. M. Knudsen, Ann. Phys-Leipzig 28, 75 - 130 (1909).

5. E. B. Arkilic, K. S. Breuer, and M. A. Schmidt, J. Fluid Mech. 437, 29-43 (2001).

6. J. Maurer, P. Tabeling, P. Joseph, and H. Willaime, Phys. Fluids 15, 2613 (2003).

7. T. Ewart, P. Perrier, I. Graur, and J. M. Meolans, J. Fluid Mech. 584, 337 - 356 (2007).

8. M. A. Gallis, and J. R. Torczynski, Phys. Fluids 24, 012005 (2012).

9. M. H. Gorji, M. Torrilhon, and P. Jenny, J. Fluid Mech. 680, 574-601 (2011).

10. T. Veltzke, and J. Thaming, J. Fluid Mech. 698, 406-422 (2012).

11. Y. Sone, Annu. Rev. Fluid Mech. 32, 779-811 (2000).

12. S. K. Dadzie, and J. M. Reese, Phys. Rev. E 85, 041202 (2012).

13. S. K. Dadzie, and J. M. Reese, Phys. Fluids 22, 016103 (2010).

14. L. García-Colin, R. Velasco, F. Uribe, Phys. Rep. 465, 149-189 (2008).

15. H. Brenner, Int. J. Eng. Sci. 47, 930-958 (2009).

16. H. Brenner, Physica A 349, 11-59 (2005).

17. I. A. Graur, J. G. Meolans, and D. E. Zeitoun, Microfluid Nanofluid 2, 64-77 (2006).

18. S. K. Dadzie, J. M. Reese, and C. R. McInnes, Physica A 387, 6079-6094 (2008).

19. K. Pong, C. M. Ho, J. Liu, Y. C. Tai, Proceedings of Application of Microfabrication to Fluid Mechanics, ASME Winter Annual Meeting, Chicago, 51-56 (1994).

20. N. Dongari, A. Agrawal and A. Agrawal, Int. J. Heat. Mass. Tran. 50, 3411-3421 (2007). 